\title{
Isoniazid Induced Liver Injury; A Case Series and Review
}

\author{
Ramatati Anusha*, Sharad Chand, Varun Lal, Devarapu Mary Sushmitha, Dhypa Sahithi Reddy, \\ Siddha Tejaswini, Savanthi Chitrahasini \\ Department of Pharmacy Practice, TVM College of Pharmacy, Ballari-583103, Karnataka, INDIA
}

Received: 16 March 2018;

Accepted: 27 May 2018

*Correspondence to:

Dr. Ramatati Anusha, PharmD,

Y. Nagesha Shastry Nagar, Kappagal Road, Ballari, Karnataka-583103, INDIA.

Email: anugracy.94@gmail.com

Copyright: (c) the author(s),publisher and licensee Indian Academy of Pharmacists. This is an open-access article distributed under the terms of the Creative Commons Attribution Non-Commercial License, which permits unrestricted non-commercial use, distribution, and reproduction in any medium, provided the original work is properly cited.

\begin{abstract}
Isoniazid is one of the most common drugs inducing liver injury, identifying and quantification of risk related to the drug is the prime to prevent and treat its occurrence. This case series tried to demonstrate the risk of drug (Isoniazid) by using the various causality assessment scales (RUCAM/CIOMS and Narnajo Algorithm). The cases taken were all males and taking the medication which on course develops the hepatotoxicity. It might be used as the literature for further study in addition similar studies are need to be carried out to understand the risk factor of drug induced liver injury.
\end{abstract}

Key words: Isoniazid, Antitubercular therapy, Drug-induced liver injury, Hepatotoxicity.

\section{INTRODUCTION}

Drug-induced liver injury (DILI) is defined as a liver injury or disease due to medications, herbs, or other toxic substances. It is the most common cause of acute liver failure in the United States (US). ${ }^{[1]}$ Directly observed treatment short course (DOTS) is the main pillar employed for the control of tuberculosis (TB) under the Revised National Tuberculosis Control Programme (RNTCP) in India. Isoniazid (INH), Pyrazinamide (PZA), and Rifampicin (RMP) used in DOTS as the main drugs are potentially Hepatotoxic and may lead to drug-induced liver injury (DILI). ${ }^{[2]}$ ATT drugs may cause Hepatotoxicity ranging from a transient asymptomatic rise in liver enzymes to acute liver failure. ${ }^{[2]} \mathrm{A}$ search in MEDLINE using the strategy "DILI or drug-induced liver injury or drug-induced liver damage or herbinduced liver injury or herb-induced liver damage or Hepatotoxicity" yielded 2196 publications in 2015 (performed on June 7th, 2016), with more than 2000 studies per year published in the past 4 years. ${ }^{[3]}$ Hepatotoxicity accounts for up to $10 \%$ of all adverse drug reaction. ${ }^{[4]}$ In a prospective study from the US, herbal and dietary supplements were found to be implicated in $10 \%$ of DILI cases. ${ }^{[4]}$

Although hepatotoxicity is a well-known side effect of INH, mortality rates remain low as reported in the literature. ${ }^{[5]}$ Other risk factors are well-confirmed including age, alcohol consumption, the concomitant administration of other hepatotoxic drugs such as acetaminophen, methotrexate, sulfasalazine, or carbamazepine, Underlying liver disease. Hepatitis B virus infection, Hepatitis $\mathrm{C}$ virus infection, HIV infection, Acetylator phenotype, $\mathrm{N}$-acetyltransferase (NAT) activity Glutathione S-transferase activity. ${ }^{[5-6]}$ It is important for clinicians and pharmacists to appropriately follow up patients, counsel them on the signs and symptoms of hepatotoxicity, and encourage them to report it. ${ }^{[5]}$ The Purpose of our study is to demonstrate the importance of INH as Potential Hepatotoxic agent. The aim of this series of Isoniazid-induced liver injury is to demonstrate the importance of isoniazid as a potential hepatotoxic agent.

\section{METHODOLOGY}

All subject suspected as drug induced liver injury by definition were taken in study. The causality assessment was done by Roussel Uclaf Causality Assessment Method (RUCAM) / Council for International Organisations of Medical Sciences (CIOMS) Scale and Naranjo Algorithm. Written informed consent was obtained from Patients and representative(s). The case studies were done according to declaration of Helsinki by maintaining the subject confidentiality.

\section{CASE SERIES \\ CASE 1}

A 50-year male patient presented in the male medical ward with the complaints of loss of appetite, generalized weakness and abdominal distension. The patient had insidious and gradually progressive course of complaints over the time of 20 days. He had a past medical history of Tuberculosis diagnosed 1 month back and started with CAT I (INH 300mg/day, RIF $600 \mathrm{mg} /$ day, PZA $1.5 \mathrm{gm} /$ day, EMB $800 \mathrm{mg}$ /day and pyridoxine $20 \mathrm{mg}$ /day) antitubercular therapy. Patient had story of alcohol intake since 13 year but stopped 3 month back. On admission he was febrile with temperature of $100^{\circ} \mathrm{F}$, pulse rate of 80 beats per minute, and blood pressure of $120 / 70 \mathrm{~mm}$ of $\mathrm{Hg}$. Physical examination revealed soft and tender uniformly distended abdomen with palpable hepatomegaly. On laboratory examination $\mathrm{X}$ rays shows bilateral diffuse opacities, and diagnosed as a case of pulmonary tuberculosis. Prothrombin time - $13.28 \mathrm{sec}$, INR- 1.2, aPTT- 26.4, USG revealed the moderate hepatomegaly with dilated peri-portal collaterals and ascitis. The liver function test parameters were markedly elevated i.e. AST- 89 U/L, ALT- $167 \mathrm{U} / \mathrm{L}$, ALP- $145 \mathrm{U} / \mathrm{L}$, and Total bilirubin was found to be $1.4 \mathrm{mg} / \mathrm{dl}$. Hepatic serological panel of test were negative hence the patient was suspected as the isoniazid induced liver injury and INH was discontinued during this course and modified anti-tubercular therapy with RESO Therapy ( R- Rifampicin 450mg, I- Ethambutol 800mg/day, S- Streptomycin 750mg and $\mathrm{O}$ - Ofloxacin $400 \mathrm{mg}$ ). The lab parameter improved over a period of week, patient condition improves considerably over a week and review after 1 month shows no hepatic manifestations. 


\section{Anusha, et al.: Isoniazid Induced Hepatotoxicity}

Causality Assessment of this case was established by Roussel Uclaf Causality Assessment methods/ CIOMS Score of 11 "Highly Probable" $(>8)$. This was confirmed by Naranjo algorithm scale with a score of 10, thus assessed as Definite ADR. This association by using various scales strengthen the Isoniazid induced hepatotoxicity for above patient.

\section{CASE 2}

A 60 years married male was admitted in the general medicine department of tertiary care hospital with the complaints of abdominal discomfort associated with the abdominal pain and generalized weakness gradually progressive since the period of 2 months. The patient was known case of pulmonary tuberculosis and on CAT I antitubercular therapy (INH $300 \mathrm{mg} /$ day, RIF 600mg/day, PZA 1.5gm/day, EMB $800 \mathrm{mg} /$ day). The family history and personal history were not found significant as patient was non smoker and non alcoholic. On examination the Vitals were stable and systemic examination revealed the tender hepatomegaly in right hypochondriac region and gastric region. Patient was having anaemia Hb- 9.8gm\% (Ref 13-17gm \%), Elevated Renal Parameters S. Urea- 56mg/dl (Ref $15-45 \mathrm{mg} / \mathrm{dl}$ ) S. Creatinine1.6 (Ref $0.6-1.2 \mathrm{mg} / \mathrm{dl})$, markedly elevated liver function parameters AST $111 \mathrm{U} / \mathrm{L}(\operatorname{Ref} 0-35 \mathrm{U} / \mathrm{L})$, ALT $96 \mathrm{U} / \mathrm{L}(\operatorname{Ref} 0-35 \mathrm{U} / \mathrm{L})$, ALP $136(\operatorname{Ref} 30-$ $120 \mathrm{U} / \mathrm{L}$ ), Indirect bilirubin was elevated up to $3.2 \mathrm{mg} / \mathrm{dl}$ (Ref $0.1-0.8 \mathrm{mg} /$ dl). USG shows marked hepatomegaly with coarse echotecture. The HIV I/ HIV II were negative along with HbsAg and HCV. The case was diagnosed as ATT induced hepatitis, the isoniazid was withdrawn, and the therapy was changed to modified approach i.e. (R- Rifampicin $450 \mathrm{mg}$, I- Ethambutol $800 \mathrm{mg} /$ day, S-Streptomycin $750 \mathrm{mg}$ and O- Ofloxacin $400 \mathrm{mg}$ ) which shows improvement, its rechallenging again led to the similar complaints. Pain abdomen was treated with hyoscine butyl bromide; Patient was prescribed with vitamin B6 and Tab Ursodeoxycholic acid as a hepatoprotectant and bile acid sequester salt.

Causality Assessment of this case was established by Roussel Uclaf Causality Assessment methods/ CIOMS Score of 8 "Probable" (6-8). This was supported by Naranjo algorithm scale with a score of 8 , thus assessed as probable ADR. Additionally, the temporal association is strongly suggestive of isoniazid-induced hepatitis.

\section{CASE 3}

A 38-year married male was admitted in the general medicine department of tertiary care teaching hospital with the complaints of yellowish discoloration of eyes for 45 days associated with weakness of both upper and lower limbs. Patient also complaints of loss of appetite and dull pain in the chest near stomach and complaints of loss of taste and pain in throat while swallowing. The patient was known case of immunodeficiency virus positive on TLE (Tenofovir, Lamivudine and Efavirenz) regimen and patient is also known case of pulmonary tuberculosis and on CAT I antitubercular therapy (INH $300 \mathrm{mg} /$ day, RIF $600 \mathrm{mg} /$ day, PZA $1.5 \mathrm{gm} /$ day, EMB $800 \mathrm{mg} /$ day) as revised national tuberculosis control programs guidelines. Patient was smoker and alcoholic but left since diagnosed with IDV. On examination the patient was poorly built, and nourished vitals measure were stable and normal, systemic examination revealed the non-tender hepatomegaly with oral thrush. Patient was having anaemia $\mathrm{Hb}-8.3 \mathrm{gm} \%$ (Ref $13-17 \mathrm{gm} \%$ ), abnormally elevated liver function parameters AST 1014 U/L (Ref 0-35U/L), ALT 997 U/L (Ref 0-35U/L), ALP 263 (Ref 30-120U/L), the ratio of ALT was increased by 30 compare to ULN as per RUCAM/CIOMS Scale. The HIV I was positive but $\mathrm{HbsAg}$ and HCV were normal. The case was suspected as antiretroviral or antitubercular induced hepatitis, the isoniazid was withdrawn as a suspected drug and the therapy was changed to modified approach i.e. (R- Rifampicin $300 \mathrm{mg}$, I- Ethambutol 600mg/day, S- Streptomycin 500mg) which shows gradual reduction in abnormal liver function parameters over a period of 60days. The patient was prescribed with iron and folic acid supplement, Inj.
Thiamine 100mg in $25 \%$ Dextrose, Tab Ursodeoxycholic acid 300mg, and topical miconazole paint for oral thrush.

Causality Assessment of this case was established by Roussel Uclaf Causality Assessment methods/ CIOMS Score of 7 "Probable" (6-8). This was supported by Naranjo algorithm scale with a score of 6 , thus assessed as probable ADR. Additionally, the temporal association is strongly suggestive of isoniazid-induced hepatitis.

\section{DISCUSSION}

Liver injury is frequently encountered in the usual therapeutic doses of isoniazid but its actual incidence and occurrence is still uncertain. There are various mechanism involved in the mechanism of liver injury associated with drugs but the definite mechanism is not clearly understood in idiosyncrasy. DILI is ascribed with more than 1000 drugs. ${ }^{[7]}$ Antitubercular drugs were major cause of liver injury accounting for $58 \%$ of all cases, followed by antiepileptic drugs, which accounted for $11.2 \%$ of liver injury. similar studies were carried out in USA and Europe where antimicrobials were major cause for liver injury. ${ }^{[8]}$ An increase of serum ALT to more than 3 fold from ULN should be followed by repeat testing within 48-72 $\mathrm{h}$ and the drug should be ruled out and discontinued. ${ }^{[9]}$ From the patients who developed drug induced liver injury almost $50 \%$ of patients develop due to the antitubercular therapy, Hence ATT should be considered for universal liver monitoring particularly during the first 8 weeks of treatment. ${ }^{[10]}$ Further similar study may clearly suggest the importance of routine test of liver function test of the patient on ATT.

\section{CONCLUSION}

All the patients in the study with isoniazid induced hepatotoxicity were males and all are on the therapy of isoniazid since they were diagnosed with the pulmonary tuberculosis. Finding the main cause and ruling out the definite drug to induce hepatotoxicity is mainstay in preventing and managing any drug related diseases. The toxicity of medication can greatly impact the disease outcome and patient quality of life. Thus this type of case series highlighting the drug induced injuries adds on to further literature on drug induced conditions.

\section{ACKNOWLEDGEMENT}

We want to thank our Principal Prof. Dr. Manjunath V Jali and HOD of Pharmacy Practice Prof. Dr. H. N Girish for their guidance and support.

\section{ABBREVIATIONS}

RUCAM: Roussel Uclaf Causality Assessment Method; CIOMS: Council for International Organisations of Medical Sciences; DILI: Drug Induced Liver Injury; DOTS: Directly Observed Treatment Shortcourse; TB: Tuberculosis; ATT: Antitubercular therapy; INH: Isoniazid; USG: Ultrasonography; INR: International Normalized Ratio; aPTT: Activated Partial Thromboplastin Time; ADR: Adverse Drug Reactions; ULN: Upper Limit of Normal.

\section{CONFLICT OF INTEREST}

Nil.

\section{REFERENCES}

1. Gayam V, et al. Drug-Induced Liver Injury: An Institutional Case Series and Review of Literature. 2018;6:1-7.

2. Saha A, Shanthi M, Winston AB, Das S, Kumar A, Michael JS, et al. Prevalence of Hepatotoxicity From Antituberculosis Therapy: A Five-Year Experience From South India J Prim Care Community Healt. 2016;7(3)171-4.

3. Raschi E, De Ponti F. Drug-induced liver injury: Towards early prediction and risk Stratification. World J Hepatol. 2017;9(1):30-7. 
4. Sait $\mathrm{M}$, et al. Drug- and herb-induced liver injury: A case series from a single Center. Turk J Gastroenterol. 2014;25(1):41-5.

5. Kabbara WK, Sarkis AT, Saroufim PG. Acute and Fatal Isoniazid-Induced Hepatotoxicity: A Case Report and Review of the Literature. Case Rep Infect Dis. 2016; 2016:3617408.

6. Sharma SK, Mohan A. Antituberculosis Treatment-Induced Hepatotoxicity: From Bench to Bedside. Medicine Update. 2005;479-84.

7. Bohm N, et al. Case Report and Cohort Analysis of Drug-Induced Liver Injury Associated with Daptomycin. Antimicrob Agents Chemother. 2014;58(8):4902-3.
8. Devarbhavi H, Dierkhising R, Kremers WK, Sandeep MS, Karanth D Adarsh CK. Single-Center Experience with Drug-Induced Liver Injury from India: Causes, Outcome, Prognosis, and Predictors of Mortality. Am J Gastroenterol. 2010;105(11):2396-404.

9. Guidance for Industry Drug-Induced Liver Injury: Premarketing Clinical Evaluation. Available from: http://www.fda.gov/BiologicsBloodVaccines/ GuidanceComplianceRegulatoryInformation/Guidances/default.htm

10. Abbara, et al. Drug-induced liver injury from Antitubercular treatment: A retrospective study from a large TB centre in the UK. BMC Infectious Diseases. 2017;17(1):231.

Cite this article as: Anusha R, Chand S, Lal V, Sushmitha DM, Reddy DS, Tejaswini S, Chitrahasini S. Isoniazid Induced Liver Injury; a Case Series and Review. J Pharm Pract Community Med. 2018;4(2):128-30. 